\title{
THREE RESULTS FOR LOCALLY COMPACT GROUPS CONNECTED WITH THE HAAR MEASURE DENSITY THEOREM
}

BRUNO J. MUELLER

In [6] I have given a generalization of the Lebesgue measure density theorem to the Haar measure on a locally compact group. A slight modification of the argument given there yields the following:

Theorem A. Let $A$ be a $\sigma$-bounded subset and $\mu$ a left-invariant Haar measure of a locally compact group $G$. Then there exists a sequence $U_{n}$ of bounded measurable neighborhoods of the unit $e$ of $G$ such that $\mu^{*}\left(A \cap U_{n} x\right) / \mu\left(U_{n} x\right) \rightarrow 1, n \rightarrow \infty$ for almost all $x$ out of a measurable cover of $A$. The sequence $U_{n}$ may be chosen out of a given basis of bounded measurable neighborhoods and simultaneously for a countable family of sets $A$.

1. A. Beck, H. H. Corson and A. B. Simon [1] have considered the inner points of a product $A B$ of two subsets of a locally compact group. They prove that $\operatorname{int}(A B)$ is not empty whenever $\mu(A)>0$, $\mu^{*}(B)>0$. The following theorem shows that $\operatorname{int}(A B)$ is quite large.

TheOREM 1. Let $A, B$ be $\sigma$-bounded subsets of a locally compact group $G, A$ measurable and $C$ a measurable cover of $B$. Then there exist subsets $A^{*}, C^{*}$ of $A, C$ equal in measure such that $A^{*} C^{*} C \operatorname{int}(A B)$.

Proof. By Theorem A we choose a sequence $U_{n}$ of compact neighborhoods of $e$ for $A^{-1}$ and $B$. We get

$$
\frac{\mu\left(A^{-1} a \cap U_{n}\right)}{\mu\left(U_{n}\right)}=\frac{\mu\left(A^{-1} \cap U_{n} a^{-1}\right)}{\mu\left(U_{n} a^{-1}\right)} \rightarrow 1, \quad \frac{\mu^{*}\left(B b^{-1} \cap U_{n}\right)}{\mu\left(U_{n}\right)} \rightarrow 1
$$

for almost all $a \in A, b \in C$. Let $A^{*}, C^{*}$ be the sets of those $a \in A$, $b \in C$ for which these limits are actually 1 . Suppose $a \in A^{*}, b \in C^{*}$ and $a b \notin \operatorname{int}(A B)$. For every neighborhood $V$ of $e$ there exists $x \in V$, $a x b \notin A B$. Consequently $A^{-1} a x \cap B b^{-1}=\varnothing$ and

$$
\begin{aligned}
\mu^{*}\left(U_{n} V\right) & \geqq \mu\left(A^{-1} a x \cap U_{n} V\right)+\mu^{*}\left(B b^{-1} \cap U_{n} V\right) \\
& \geqq \mu\left(A^{-1} a x \cap U_{n} x\right)+\mu^{*}\left(B b^{-1} \cap U_{n}\right) \\
& =\mu\left(A^{-1} a \cap U_{n}\right) \Delta(x)+\mu^{*}\left(B b^{-1} \cap U_{n}\right)
\end{aligned}
$$

If $V$ is a small enough neighborhood of $e$, it follows that $\Delta(x)$ is arbitrarily close to 1 , and that $\mu^{*}\left(U_{n} V\right)$ is arbitrarily close to $\mu\left(U_{n}\right)$

Received by the editors January 15, 1965. 
for any fixed $n$ since each $U_{n}$ is compact and $\mu$ is regular. Thus, for each $n$,

$$
\mu\left(U_{n}\right) \geqq \mu\left(A^{-1} a \cap U_{n}\right)+\mu^{*}\left(B b^{-1} \cap U_{n}\right) .
$$

However, for $n$ large enough, both

$$
\frac{\mu\left(A^{-1} a \cap U_{n}\right)}{\mu\left(U_{n}\right)} \text { and } \frac{\mu^{*}\left(B b^{-1} \cap U_{n}\right)}{\mu\left(U_{n}\right)}
$$

are greater than $1 / 2$, yielding the contradiction

$$
\mu\left(U_{n}\right) \geqq \mu\left(A^{-1} a \cap U_{n}\right)+\mu^{*}\left(B b^{-1} \cap U_{n}\right)>\mu\left(U_{n}\right) .
$$

REMARK. The theorem yields explicit estimates of the measure of $\operatorname{int}(A B)$. For $\mu(A)>0$ and $a \in A^{*}$ we have

$$
\mu(\operatorname{int}(A B)) \geqq \mu^{*}\left(A^{*} C^{*}\right) \geqq \mu\left(a C^{*}\right)=\mu\left(C^{*}\right)=\mu^{*}(B) .
$$

Other estimates for $\mu(A B)$ (e.g. M. Kneser [4]) can be used in the same manner.

2. On the basis of Theorem A I have studied e-joint semigroups in locally compact groups [7]; these are semigroups $N$ such that $\mu_{*}(N \cap U)>0$ for all neighborhoods $U$ of $e$. They have the fundamental property that their boundary $\bar{N} \backslash \operatorname{int}(N)$ is of measure zero.

M. Zorn [2, p. 157] has proved the following:

THEOREM B. If $N$ is a semigroup in a topological group which is of the second category at $e$ and satisfies the condition of Baire, then int $(N)$ $=\operatorname{int}(\bar{N})$ and $\operatorname{int}(N)$ is dense in $N$.

Theorem 2. $A$ semigroup $N$ in a locally compact group $G$ is e-joint if and only if it is of the second category at $e$ and satisfies the condition of Baire.

Proof. In a locally compact group $G$ every nonempty open set $A$ is of the second category. For suppose $A=\bigcup_{i-1}^{\infty} A_{i}, A_{i}$ nowhere dense in $G$. For each $i$ and each nonempty open set $U$ there exists a nonempty bounded open set $V$ with $\bar{V} \subset U, \bar{V} \cap A_{i}=\varnothing$. Starting with $V_{0}=A$ we construct a sequence of nonempty bounded open sets $V_{n}$, $n \geqq 1$ with $\bar{V}_{n} \subset V_{n-1}, \bar{V}_{n} \cap A_{n}=\varnothing$. They form a filter-basis on the compact set $\bar{V}_{1}$ having a cluster point $x \in \bar{V}_{1} \subset V_{0}=A, x \in \bar{V}_{n}, n \geqq 1$. This implies the contradiction $x \notin A_{n}, n \geqq 1$ and $x \notin A$.

If $N$ is $e$-joint, $\mu(\operatorname{int}(N) \cap U)=\mu(N \cap U)>0$ for all open neighborhoods $U$ of $e$; hence $\operatorname{int}(N) \cap U$ is of the second category, and so is $N$ at $e$. Further, as the measure of the boundary of $N$ is zero, the boundary has empty interior; and that implies the condition of Baire. 
Conversely, if the conditions of Theorem B hold for $N$, we have $e \in \bar{N}=(\operatorname{int}(N))^{-}$, therefore $\operatorname{int}(N) \cap U \neq \varnothing$ for all open neighborhoods $U$ of $e$ and $\mu_{*}(N \cap U) \geqq \mu(\operatorname{int}(N) \cap U)>0$, so $N$ is $e$-joint.

3. A subadditive function $f$ on a locally compact group $G$ is a function whose values are real or $\pm \infty$ and which satisfies $f(x y) \leqq f(x)$ $+f(y) .{ }^{1}$ Such functions have been studied by E. Hille and M. Zorn [3], [2], R. A. Rosenbaum [8] and others, mainly in connection with semigroups.

With a subadditive function $f$ there are associated the functions $f_{*}(x)=\lim \inf _{y \rightarrow x} f(y)$ and $f^{*}(x)=\lim \sup _{y \rightarrow x} f(y)$ which are subadditive, lower resp. upper semicontinuous and satisfy $f_{*} \leqq f \leqq f^{*}$.

THEOREM 3. Let $f$ be a subadditive function on a locally compact group $G$ satisfying $\lim \inf _{x \rightarrow \infty} f^{*}(x) \leqq 0$. Then $f$ is continuous almost everywhere.

Proof. Let $R$ be the additive group of the real numbers and $N \subset G \times R$ the set $\{(x, \alpha) \mid f(x) \leqq \alpha\} . N$ is a semigroup, $\operatorname{int}(N)$ $=\left\{(x, \alpha) \mid f^{*}(x)<\alpha\right\}$ and $\bar{N}=\left\{(x, \alpha) \mid f_{*}(x) \leqq \alpha\right\}$. The condition $\lim \inf _{x \rightarrow 0} f^{*}(x) \leqq 0$ implies (and is actually equivalent to) the $e$-jointness of $N$. Hence the boundary of $N$ is of measure zero, and this measure is equal to $\int_{\theta}\left(f^{*}(x)-f_{*}(x)\right) d \mu$. Consequently $f_{*}(x)=f^{*}(x)$ almost everywhere.

REMARKs. If $\lim \inf _{x \rightarrow e} f^{*}(x)>0, f$ may be discontinuous everywhere.

$\lim \inf _{x \rightarrow \infty} f^{*}(x) \leqq 0$ implies $=0$ or $=-\infty$. If it is $=-\infty, f$ may be described as follows: There exists an $e$-joint semigroup $N$ in $G$ (whose boundary is of measure zero) such that $f(\operatorname{int}(N))=-\infty, f\left(\operatorname{int}\left(N^{\prime}\right)\right)$ $=+\infty$.

\section{REFERENCES}

1. A. Beck, H. H. Corson and A. B. Simon, The interior points of the product of two subsets of a locally compact group, Proc. Amer. Math. Soc. 9 (1958), 648-652.

2. E. Hille, Functional analysis and semi-groups, Amer. Math. Soc. Colloq. Publ. Vol. 31, Amer. Math. Soc., Providence, R. I., 1948.

3. E. Hille and M. Zorn, Open additive semi-groups of complex numbers, Ann. of Math. 44 (1943), 554-561.

4. M. Kneser, Summenmengen in lokalkompakten Abelschen Gruppen, Math. Z. 66 (1956), 88-110.

5. E. J. McShane, Images of sets satisfying the condition of Baire, Ann. of Math. 51 (1950), 380-386.

6. B. Mueller, Eine Verschaerfung fuer Abschaetzungen von Summenmengen in lokalkompakten Gruppen, Math. Z. 78 (1962), 199-204.

7. - Halbgruppen und Dichteabschaetzungen in lokalkompakten abelschen Gruppen, J. Reine Angew. Math. 210 (1962), 89-104.

8. R. A. Rosenbaum, Sub-additive functions, Duke Math. J. 17 (1950), 227-247.

University of Mainz, Germany

1 With $(+\infty)+(-\infty)=+\infty$. 